\title{
ANALYSIS OF PACING ALGORITHMS FOR PROACTIVE CHAT CAMPAIGN
}

\author{
Nikolay Korolev \\ Herbert Ristock \\ Architecture Team \\ Genesys Telecommunications Laboratories, Inc. \\ 2001 Junipero Serra Blvd. \\ Daly City, CA, USA \\ \{nikorolev,herbert.ristock\}@gmail.com
}

\begin{abstract}
Customer Experience $(\mathrm{CX})$ centers observe an increase of chat traffic compared to other media types such as voice or email when serving customers. Similar to voice there are regular inbound chat interactions opened by customers, and in addition many CX centers widely use proactive chat campaigns to improve their services. Design and implementation share many similarities with voice outbound campaigns, in particular the use of pacing algorithms (PA). In this paper we describe some specifics of chat proactive customer engagement working in blended mode with proactive and inbound interactions processed by the same group of agents. We suggest a new pacing algorithm and present simulation results comparing its efficiency with other existing algorithms.
\end{abstract}

Keywords: proactive campaign, pacing algorithm, contact center, CX.

\section{INTRODUCTION}

CX centers use various pacing algorithms primarily for voice outbound campaigns, as described and analyzed in (Anisimov, Korolev and Ristock 2008; Amaral and Vital 2014). This paper expands those solutions to proactive chat campaigns, which bear many similarities but also some differences.

Internet web services are increasingly important in people's daily life, and use of text messaging has become very common including consumer-to-business communication with $\mathrm{CX}$ centers. When browsing a company's website there is often a static click-to-chat button displayed. When engaging through this button a chat session is generated and placed into the company's CX center queue. Then the system starts interacting with the customer, either offering self-service help or asking questions in order to find the appropriate agent for assisted service. This process of asking and answering questions could be organized by a special software program called chatbot. Its behavior is similar to IVR (interactive voice response) functionality for inbound voice calls, and dialogs might be very complex. Usually at the end of this initial conversation the system decides whether appropriate self-service is available or there is a need for agent assisted service. In case of self-service the interaction is served and finished without engagement of an agent. For assisted service the customer begins to wait for an agent. If waiting time in the queue is too long, the customer might abandon the session. This is a regular customer initiated inbound chat interaction flow, similar to inbound voice calls (Gans, Koole and Mandelbaum 2003).

Leveraging capabilities of web technologies companies can implement also proactive chat campaigns, where an invitation for a chat conversation is displayed dynamically at the company's web site depending 
on various criteria, including business relevance but also ability to serve the interaction in a timely fashion. When a visitor opens a website, at a certain moment a popup message might be displayed inviting to have a chat conversation with an agent. We will call this message an invitation or just an invite. The visitor might either accept or reject the invitation. We will refer to accepted invites as successfully answered or just answered. The visitor might not react immediately, having a pending status where the invite is neither answered nor rejected. Usually there is a timeout after which a pending invite is automatically closed. The number of open pending invites is an important statistic that is used in many pacing algorithms. If an invite is answered by a visitor, the system converts it to a chat session and places it into the queue to wait for the next available agent. The further processing of the proactive chat session is similar to an inbound chat described above. The percentage of accepted invites in relation to all generated invites is called hit rate. A typical hit rate for chat proactive campaigns is about $10 \%$, while for voice outbound campaigns the value is typically about $30 \%$. This difference might be explained by the fact that when receiving a chat invite a customer knows the sender and subject of the invite, which isn't usually the case for voice calls. This enables more qualified decision making on whether or not to accept the invite. The considerable difference in hit rates is relevant for proper pacing. A lower hit rate requires more invites to be generated. The control of a campaign becomes more difficult because the outcome is less predictable and requires more sophisticated pacing algorithms to improve efficiency. If a chat interaction stays in the queue longer than patience time (time willing to wait in the queue) of a customer, the chat becomes abandoned. The abandonment rate, which is the percentage of abandoned chats to all chats placed into the queue, should not be too high. A typical upper limit for abandonment rate is between $3 \%$ and $5 \%$. Another important parameter of a proactive campaign is called agent busy factor or just busy factor. Basically, it is the agents' utilization level which shows what percentage of time on average the agents are busy processing chat sessions. Here we will consider blended campaigns that have both types of chats - proactive and inbound, and an agent can be assigned only one chat session at a time.

This paper provides a performance analysis of several pacing algorithms for blended chat campaigns. Using simulation results it will be shown that none of the previously suggested in (Anisimov, Korolev and Ristock 2008) algorithms works adequately for the blended environment. A new pacing algorithm will be introduced. It does not require to collect any statistics based on historical data. That is why it is called nonparametric. This algorithm will significantly improve the performance of proactive chat campaigns for relatively small agent groups with considerable amount of inbound chat traffic.

\section{THE MODEL, ASSUMPTIONS AND PROBLEM STATEMENT}

Let's consider the model of a proactive chat campaign in the blended environment. It can be presented as a queuing system including the following major elements: a group of agents, a chat (invites) generator, a queue. This queue contains both proactive and inbound chat sessions. The chat invites generator is controlled by PA. The task of a pacing algorithm is to predict how many invites need to be sent to visitors on a website at a certain moment of time in order to maximize the busy factor of agents while keeping the abandonment rate below a predefined threshold. The campaign structure is presented on Figure 1.

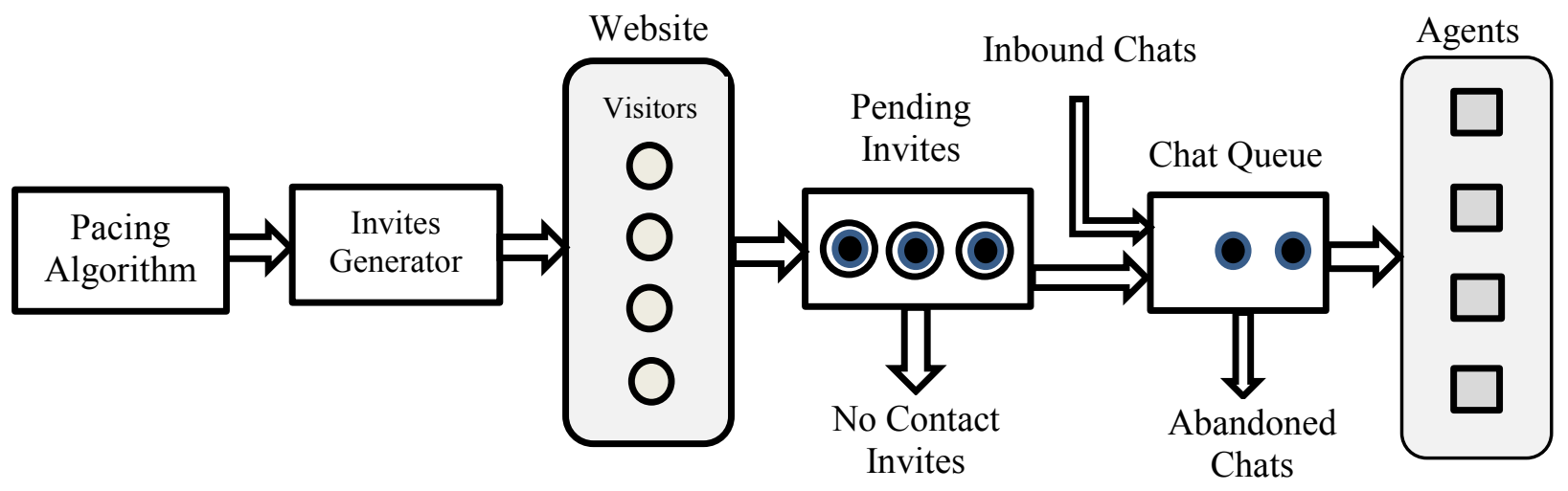


Figure 1. Proactive blended chat campaign.

In the campaign model we assume that a pacing algorithm (PA) makes predictions at the beginning of each time slot. If the time slot duration is $\mathrm{T}$ which for instance could equal 1 second, the PA decisions are made at time values $0,1,2, \ldots$, and the $n$-th time slot is the interval $[n T,(n+1) T]$. All campaign variables including queue length $Q(n)$ are computed at times $t(n)=n T$. We will introduce the other variables when describing particular PAs in the next sections.

We assume that the following campaign parameters do not depend on time: $h$ - hit rate, $\omega_{a}, \omega_{n}-$ mean values of durations of the pending invites until positive answer and no contact response from a visitor respectively, $\tau$ - agent chat average handling time (AHT), $\theta_{p}, \theta_{i n}$ - patience time for proactive and inbound chats respectively, $\lambda_{i n}$ - arrival rate for inbound chats, $N$ - total number of agents in a group. However, the assumption that all these parameters are fixed can be relaxed. Simulations show that relatively slow changes do not lead to significant degradation of most PAs presented in this paper.

It is supposed that campaign objects such as visitors, invites, chat sessions and agents in a campaign are homogeneous and independent, with equal corresponding stochastic behavioral properties. The inbound chats are considered being a Poisson process with intensity $\lambda_{i n}$.

This leads to a problem statement for the PA design to properly estimate the number of invites $I(n)$ that the system should generate at times $t(n)$. In this paper we will consider the abandonment rate $A$ as an optimization constraint for all investigated PAs, that means that actual abandonment rate $A(n)$ shall not exceed a predefined threshold $A^{*}$. The optimization goal is to maximize the busy factor $B$.

Since several PAs will be considered in this paper, it is necessary to formulate the criteria to compare their efficiency. Let's consider two algorithms PA1 and PA2 in a long run, assuming that they have the same campaign model parameters. We are interested in the behavior of performance characteristics (abandonment rate and busy factor) of a campaign after several hours of continuous work, let say at time $t^{*}=n^{*} T \geq 10$ hours. Supposed that the abandonment rates $A_{1}\left(n^{*}\right), A_{2}\left(n^{*}\right)$ for all investigated algorithms are below threshold $A^{*}$, then the algorithm with highest busy factor $B\left(n^{*}\right)$ is considered the best. If the abandonment rate of a certain PA is higher than $A^{*}$, it is considered being inadequate and excluded from comparison because it does not satisfy the optimization constraint of $A\left(n^{*}\right)$ being less than $A^{*}$.

\section{KNOWN PACING ALGORITHMS}

In this section we will describe the PAs that were introduced in (Anisimov, Korolev and Ristock 2008) and explain why they are not fully applicable to blended proactive campaigns. More details will be provided in section 5 where they are compared with a new Nonparametric PA.

\subsection{Progressive}

For the Progressive PA the number of invites $I$ is calculated by the following formula, here and thereafter we will omit the reference to the $n$-th time slot

$$
I=N_{r}-M_{p}-Q
$$

where $N_{r}$ - number of ready agents, $M_{p}$ - number of pending invites, $Q$ - queue length, comprising both proactive and inbound chats. Obviously, if the expression on the right side of (1) is negative, then the value of $I$ is zero. Theoretically the queue length should be zero when there is at least one ready agent in a group. However, in practice this might not be always the case due to certain delays in the system and some preliminary processing of chats in the queue. 
This is a rather simple and very conservative algorithm. Usually it is used to minimize the abandonment rate. For pure proactive campaigns without inbound traffic it guarantees a zero value for the abandonment rate. But the drawback is a low busy factor for low values of the hit rate.

\subsection{Predictive}

As pointed out in (Anisimov, Korolev and Ristock 2008), this pacing method relies on the Erlang B model from queuing theory, with notation $M / G / N / N$ of the corresponding queuing system (Kleinrock 1975). The main idea is to create a stable flow of invites with intensity $\lambda_{p}$, so that the estimated abandonment rate of the total chats flow with intensity $\lambda=\lambda_{p} h+\lambda_{\text {in }}$ will be equal to the threshold $A^{*}$.

Let's denote service rate as $\mu=1 / \tau$ and traffic offer $\rho=\lambda / \mu$. According to the Erlang loss formula the optimal value $\rho^{*}$ of $\rho$ can be found from the following nonlinear equation

$$
\frac{\rho^{N} / N !}{\sum_{i=0}^{N} \rho^{i} / i !}=A^{*}
$$

The value of $\lambda_{p}$ can be obtained from (2) and the expression: $\lambda_{p}=\left(\rho^{*} \mu-\lambda_{i n}\right) / h$. Then the number of invites per time slot is $I=\lambda_{p} T$. Even though this value is virtually always fractional, whereas the algorithm is expected to provide a non-negative integer number, several approaches can be suggested. One possibility is to randomly generate the number of invites so that their expected value will be equal to $\lambda_{p} T$. Another solution is to round $I$ and add the remainder to the value for the next time slot. Here we assume that all necessary parameters such as hit rate $h$, AHT and inbound chats arrival rate $\lambda_{\text {in }}$ can be estimated by the system based on historical data.

One of the advantages of this algorithm is that it allows to analytically calculate the agents busy factor for a given value of the target abandonment rate $A^{*}$. From practical point of view, it is very useful to know in advance what to expect from the algorithm in particular circumstances. This property is described in more details in paper (Anisimov, Korolev and Ristock 2008). Here we would like to mention that the performance of this algorithm significantly depends on the size of agent group. It provides a relatively high busy factor for large agent groups. On the other hand, its performance degrades for small agent groups. For example, in a pure proactive scenario, when $N=100, A^{*}=5 \%$, a typical value for busy factor is around $90 \%$; while for $N=20, A^{*}=5 \%$, the busy factor usually does not exceed $70 \%$. The situation does not improve by incorporating inbound traffic to the campaign. This small group problem triggered the introduction of more sophisticated PAs.

\subsection{Super Progressive}

This algorithm can be considered as an enhancement of the Progressive algorithm. It suggests generating more invites than the current value of ready agents minus number of pending invites and the queue length as for the Progressive algorithm. Though this algorithm was suggested in paper (Anisimov, Korolev and Ristock 2008), we will provide a more detailed explanation of the calculations and also slightly change its original version.

At the beginning of the $n$-th time slot this algorithms takes just two parameters: $k$-number of ready agents, $h$ - hit rate. Since each invite is assumed to be independent of others and has the same probability $h$ of being answered by a visitor, we have a classical Bernoulli process with two outcomes (Chiasson 2013). When the system sends $m(m \geq k)$ invites, the number of answered invites $\xi$ has a binomial distribution $B(m, h)$. Let's suppose that there are no pending invites and the queue is empty. All answered invites that exceed the number of ready agents will be abandoned when they will become chat sessions. The probability $P(m, h, k, i)$ of $i$ abandoned chats is the probability of the event $\{\xi=k+i\}$

$$
P(m, h, k, i)=\left(\begin{array}{c}
m \\
k+i
\end{array}\right) h^{k+i}(1-h)^{m-k-i}, i=\overline{0, m-k}
$$


This gives the probability distribution of the abandonment rate in this situation. Its values are $i /(k+i)$ with corresponding probabilities $P(m, h, k, i)$ from (3). The formula for the mean value $A(m, h, k)$ of this random variable is

$$
A(m, h, k)=\sum_{i=1}^{m-k} \frac{i}{k+i} P(m, h, k, i)
$$

Using formula (4) the final value of the number of invites $I$ for $n$-th time slot equals to a maximal $m^{*}$ that satisfies the inequation $A(m, h, k) \leq A^{*}$ minus number of pending invites and the queue length. This is actually the difference compared to the corresponding algorithm in (Anisimov, Korolev and Ristock 2008), where it was suggested that a new set of invites has to be sent only when the number of pending invites is zero and the queue is empty. The current approach relaxes this requirement and allows to send invites even if at least one of these two parameters is positive, and finally it gives $I=m^{*}-M_{p}-Q$.

This algorithm outperforms both Progressive and Predictive for pure proactive campaigns. Usually it has a noticeably lower abandonment rate than the target $A^{*}$. Even in presence of inbound chats it behaves fairly until arrival rate becomes relatively large. The actual abandonment rate starts to significantly exceed the target value $A^{*}$ only when the average duration between incoming inbound chats is equal or less the mean value $\omega_{a}$ of pending invites duration until being answered. We will see this drawback when the simulation results will be discussed. On the other hand, there is no surprise in such performance degradation. Indeed the algorithm does not directly take inbound traffic into consideration. Most probably it is possible to update this algorithm to fix this problem. Some attempts were done by the authors of this paper. But the behavior of these patches was not stable and required either very complex calculations or introducing some additional parameters that need to be manually fine-tuned. As an alternative we propose a new and completely different approach of the pacing algorithm that improves performance of blended proactive campaigns.

\section{NONPARAMETRIC PACING ALGORITHMS}

This algorithm is an extension of the pacing methods applied to so called knowledge workers (KW) described in referenced papers (Stolyar et al. 2008; Pang and Stolyar 2016). Knowledge workers are temporary CX center agents who are engaged into an inbound campaign on-demand. Such campaigns do not have a fixed set of agents. The goal of PA is to invite the proper number of KWs to safely process incoming traffic of phone calls or other types of interaction. It appeared that a similar algorithm can be implemented in a proactive campaign considered in this paper.

Unlike the three PAs described above this one does not require to estimate model parameters like hit rate or AHT which are based on historical data. It just takes into consideration the most recent events which occurred in a campaign - therefore it is named nonparametric. The main idea is to maintain the number of pending invites $M_{p}$ to approach a certain target $M_{p}^{*}$. The precise value of this target is not known in advance. But we can use a rule of its update that will allow to keep the chat abandonment rate close to the goal $A^{*}$. According to this rule the current value of the target at the beginning of $(n+1)$-th time slot is increased by $\alpha A^{*}$ for each chat which is successfully transferred to an agent during the $n$-th time slot and is decreased by $\alpha\left(1-A^{*}\right)$ for each abandoned chat. Here $\alpha$ is a positive parameter which controls the speed of reactions on changes in pending invites. For a relatively wide range of the model parameters the value $\alpha=1$ was quite adequate. It will be used in all simulations presented in this paper.

Before presenting the algorithm itself we introduce and initialize some new parameters and auxiliary variables: $S(n)$ - a shift of the target $M_{p}^{*}(S(0)=0), Y(n)=Q(n)-k(n)$ - difference between queue length and number of ready agents, $\beta$ - parameter that controls the speed of reactions on changes of $Y(n)$. In our simulations we used $\beta=1$. Below are the steps to compute the number of invites $I(n+1)$ that has to be generated at the beginning of the $(n+1)$-th time slot:

1. Collect the following statistics related to the previous $n$-th time slot: $C_{s}(n)$-number of chats taken for service by the agents, $C_{a}(n)$ - number of chats abandoned from the queue. 
2. Update the shift: $S(n+1)=S(n)+\alpha\left(C_{S}(n) A^{*}-C_{a}(n)\left(1-A^{*}\right)\right)$.

3. Update the target number of pending invites: $M_{p}^{*}(n+1)=M_{p}^{*}(0)+S(n+1)-\beta Y(n+1)$. Here $M_{p}^{*}(0)$ is an initial estimation of the target. In the simulations it was set to zero, though more precise values can reduce the transient period.

4. Calculate number of invites: $I(n+1)=M_{p}^{*}(n+1)-M_{p}(n+1)$ which is the difference between target and real values of invites.

5. Store $S(n+1)$ for the next time slot calculations.

As we see this algorithm does not use any model parameter that requires some historical data. It is much faster than Predictive and Super Progressive algorithms. This property becomes useful when the system runs many proactive campaigns simultaneously. Most importantly, the Nonparametric pacing algorithm outperforms all others for small agent groups in blended environment. We will discuss this in the next section in more detail.

\section{SIMULATION RESULTS AND DISCUSSION}

The simulation tests were conducted in the following conditions:

1. All four types of pacing algorithms considered in this paper were tested. Each experiment is obtained as a Monte Carlo simulation by a computer program of a chat blended proactive campaign lasting 10 hours. Although several advanced simulation tools are available for researchers, we developed our own Java application. It is based on discrete-event simulation (Banks at el. 2009) which appeared to be more efficient than an agent-based approach for contact center modeling.

2. For each set of the model input parameters 8 experiments were conducted. Since these experiments are independent, they were run in separate threads on the same computer in parallel using a MapReduce programming model (Miner, Shook 2013). The results were averaged and are presented in the charts below. The standard error of the test results is less than $2 \%$.

3. All durations in the model like pending times, AHT, patience times were generated as independent and exponentially distributed random variables.

4. The model input parameters had the following values: $h=10 \%, \omega_{a}=\omega_{n}=30 \mathrm{sec}, \theta_{p}=5 \mathrm{sec}$, $\theta_{\text {in }}=1$ hour, $\tau=300 \mathrm{sec}, A^{*}=5 \%$. Inbound arrival rate $\lambda_{\text {in }}$ has been adjusted to the number of agents in order to achieve an inbound agent busy factor around $48 \%$. On Figure 3 it relates to "Inbound only" simulation results.

\subsection{Agent Group Size}

The purpose of the simulation experiments was to show dependency of abandonment rate and busy factor on the number of agents in the group. While analyzing the simulation results presented on Figures 2 and 3, an obvious question arises: which algorithm is better? Let's recall the best candidate criteria suggested above: abandonment rate below the threshold and higher busy factor. To simplify the analysis, let's split the tests into two cases:

1. small-group case: number of agents is less than 50 ,

2. large-group case: number of agents is greater or equal to 50 .

It is clear that the Nonparametric PA outperforms others in the small-group case. Only for an extremely small 5-agent group experiment it has an abandonment rate slightly higher than the goal. The nearest competitor is the Progressive algorithm which has a low abandonment rate but provides in some tests a significantly lower busy factor. The other two PAs exceed the performance goal $A^{*}$ and cannot compete for the performance leadership.

We can see from the Figures 2 and 3 that the Super Progressive algorithm is an apparent winner in the largegroup case. When the number of agents grows, its abandonment rate goes strongly down, and the busy 


\section{Korolev and Ristock}

factor is permanently the highest among others. On the other hand, there is no significant gap in busy factor between Nonparametric and Super Progressive algorithms in the large-group case. Therefore, we suggest using the Nonparametric algorithm in practice when proactive traffic becomes additional workload for an inbound agent group.

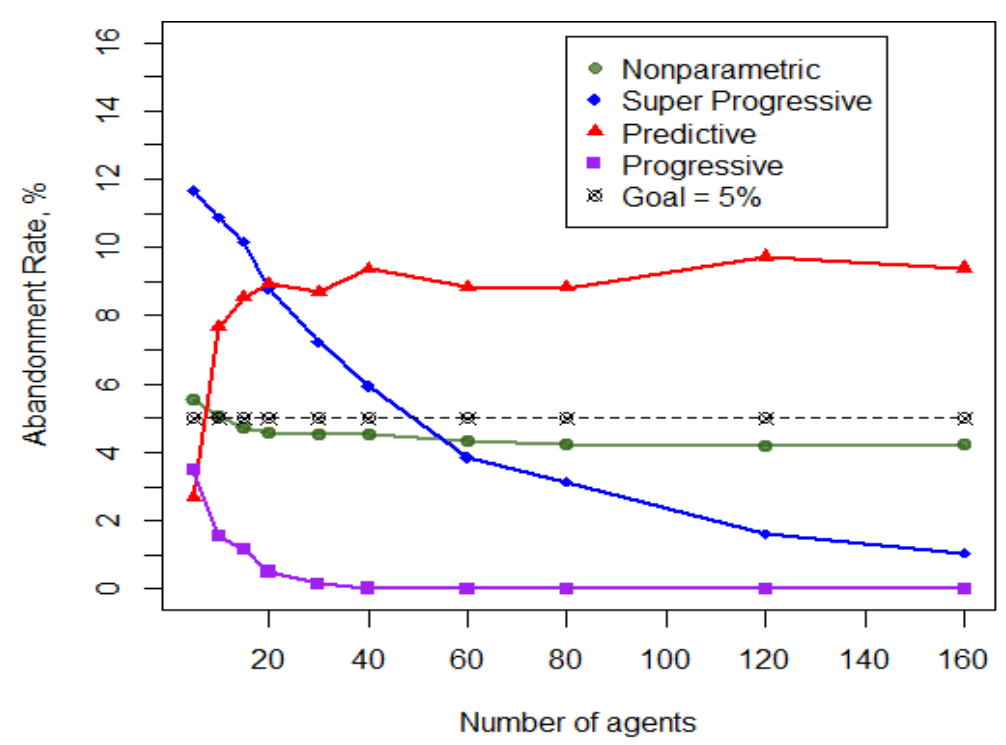

Figure 2. Abandonment rate for blended chat campaign. Agent Group Size Scenario.

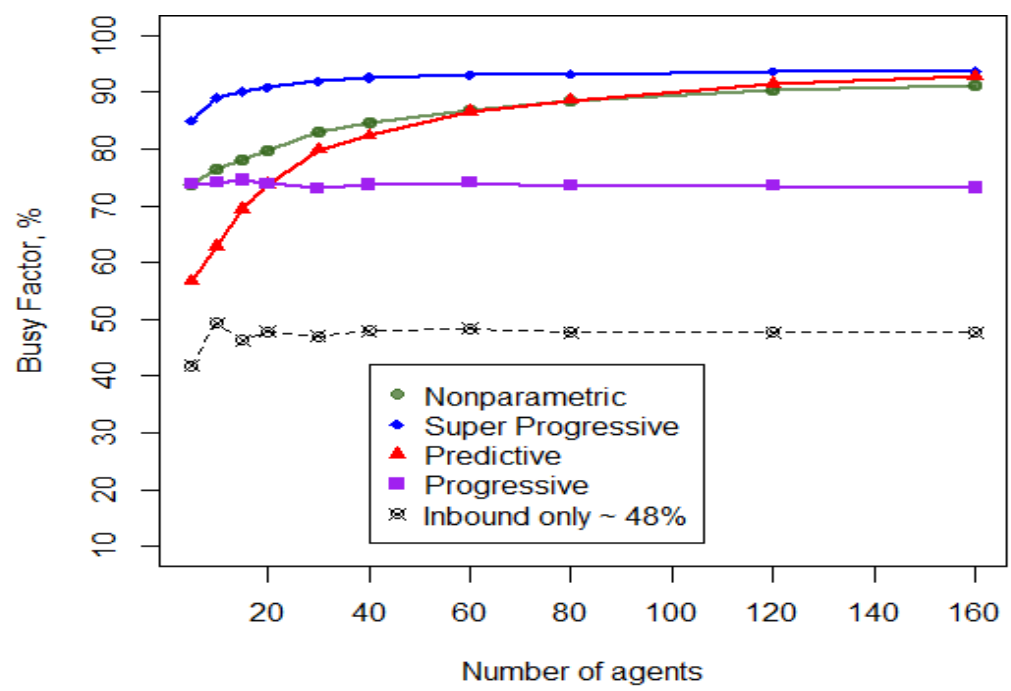

Figure 3. Busy factor for blended chat campaign. Agent Group Size Scenario.

\subsection{Inbound Traffic Portion}

This set of tests aims to analyze performance of the PAs when the portion of inbound traffic grows. From practical point of view, it is necessary to understand whether the same relation of performance characteristics of the analyzed algorithms applies also to a broader range of scenarios, when the mix of two types of chats changes from a proactive-only campaign to inbound almost complete domination. 
Figures 4 and 5 represent dependency of abandonment rate and busy factor on inbound arrival rate. In these tests the input parameter values are the same as in 5.1 tests. The number of agents was fixed, $N=20$, whereas inbound arrival rate varied from zero to the point when inbound chats covered up to $83 \%$ of agent occupancy (see "Inbound only" line on Figure 5). So, a small-group case was considered.

Firstly, let's look at a scenario when inbound traffic is either absent or relatively low, not exceeding $30 \%$ of agent occupancy with arrival rate below 0.02 chats per second as shown on Figure 5 . In this case the Super Progressive algorithm is better than others, though Nonparametric is close. Same as in the previous set of tests these both are apparent leaders.

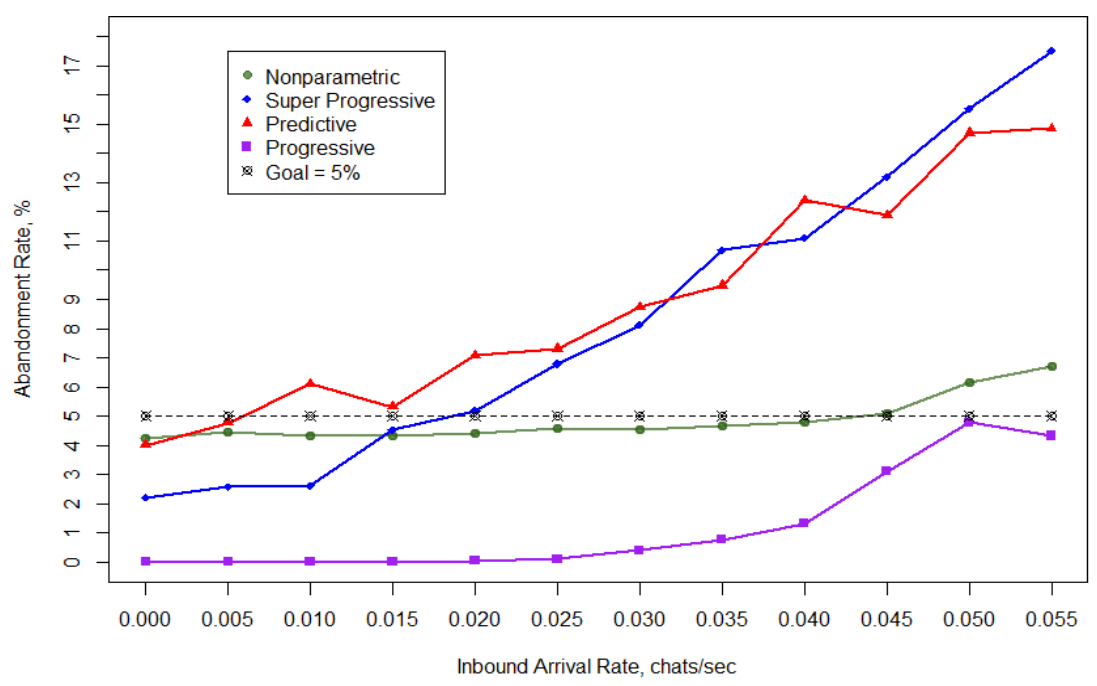

Figure 4. Abandonment rate for blended chat campaign. Inbound Traffic Portion Scenario.

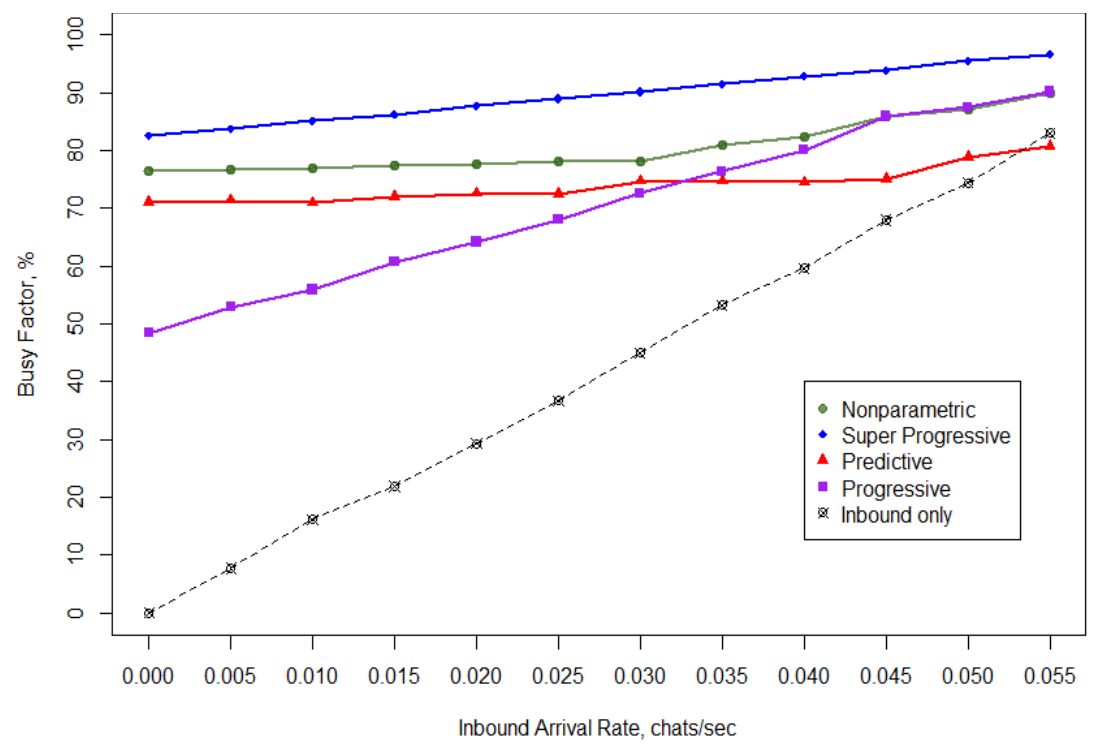

Figure 5. Busy factor for blended chat campaign. Inbound Traffic Portion Scenario.

The situation changes when the inbound arrival rate further grows. The abandonment rate for the Nonparametric algorithm remains close to the goal 5\%, while for Super Progressive it grows significantly. This renders the latter not that useful for proactive campaigns with high portion of inbound traffic. 
Interestingly, the Progressive algorithm which is usually considered an outsider, behaves as efficient as the Nonparametric for high inbound load.

\section{CONCLUSIONS AND FUTURE WORK}

The pacing algorithms play significant role in performance optimization of proactive campaigns. In this paper their use was extended to chat proactive campaigns. A new Nonparametric algorithm was introduced. The simulation results proved its higher performance in blended environment.

Web technologies overall - and the chat media in particular - offer more advanced capabilities compared to voice calls where PAs were traditionally implemented. For example, unlike regular voice inbound traffic it is technically possible to control the intensity of chat sessions flow. It can be done through special software applications that will show or hide click-to-chat buttons on the web pages according to agent availability or some other conditions and rules. Now it is possible to keep a desirable balance between inbound and proactive chats by manipulating both of them through pacing algorithms. Additionally, chat media typically allows an agent to process more than one interaction at the same time. It makes agent behavior more complex and requires significant changes of the campaign models.

Another area for pacing algorithms expansion is voice callback campaigns. On many websites there exists a click-to-call button. It allows to schedule a callback at the most convenient time for both a customer and a contact center. Once a callback is scheduled, it is necessary to use a pacing algorithm to make a corresponding outbound call at a proper time.

The pacing algorithms described in this paper have also huge opportunities for enhancements. The Super Progressive algorithm still has a chance to be better adjusted for inbound traffic. The Nonparametric algorithm requires additional research to more optimally manipulate control parameters $\alpha$ and $\beta$.

Since now we know how to handle proactive campaigns in conjunction with inbound traffic, it gives the opportunity to run several proactive campaigns against a single agent group. Moreover, there could be a mixture of proactive, inbound and some other types of activities assigned to the same agents. Also some agents might belong to several groups simultaneously. All these ideas need further analysis and implementation.

\section{REFERENCES}

Amaral, P., M. Vital. 2014. "Predictive Dialer Intensity Optimization Using Genetic Algorithms". International Journal of Machine Learning and Computing, vo. 4, No. 3, pp. 286-291.

Anisimov, N., N. Korolev, and H. Ristock. 2008. "Modeling and simulation of a pacing engine for proactive campaigns in contact center environment". In Proceedings of the 2008 Spring Simulation Multiconference, pp. 249-255. San Diego.

Banks J., J.Carson, B. Nelson, D. Nicol. 2009. Discrete-Event System Simulation. Pearson.

Chiasson J. 2013. Introduction to Probability Theory and Stochastic Processes. John Wiley \& Sons.

Gans N.,G. Koole, A. Mandelbaum. 2003. Telephone Call Centers: Tutorial, Review and Research Prospect, Manufacturing and Service Operations Management, vol. 5, No. 2, pp. 79-141.

Kleinrock L. 1975. Queueing Systems.Vol. I: Theory. John Wiley \& Sons.

Miner D., A. Shook. 2013. MapReduce Design Patterns. O’Reilly.

Pang,G., A. L. Stolyar. 2016. “A service system with on-demand agent invitations”. Queueing Systems, vol. 82 , No. 3, pp. 259-283. 
Stolyar, A.L., M.I. Reiman, N. Korolev, V. Mezhibovsky, and H. Ristock. 2018. "Pacing in Knowledge Worker Engagement”. United States Patent No. US 9,955,012.

\section{AUTHOR BIOGRAPHIES}

NIKOLAY KOROLEV is a scientific researcher at Genesys Telecommunication Laboratories (Daly City, CA, USA). He received M.Sc. in computer science and Ph.D. degree in statistics from Yaroslavl State University (Russia) in 1988 and 1995 respectively. He has wide range of research interests: statistical data analysis, pattern recognition, queuing theory, analysis and simulation of contact center activities, geographical information systems. Prior joining Genesys he worked as an associate professor at Yaroslavl State University. He has more than 10 publications and 15 patents. His email address is nikorolev@gmail.com.

HERBERT RISTOCK is a Senior Director of Software Development at Genesys Telecommunication Laboratories (Daly City, CA, USA). He studied Mathematics and holds a Ph.D. in Applied Mathematics from Leipzig University (Germany). His current research interests are related to contact center service engineering, software design, optimization and simulation. He is a member of IEEE, INFORMS. He has several work related publications and holds several patents. His email address is herbert.ristock@gmail.com. 